\title{
Transdiagnostic Processes as Mediators of Change in an Internet-Delivered Intervention Based on the Unified Protocol
}

\author{
Carmen Schaeuffele ${ }^{1}$ (1) . Jonathan Bär ${ }^{1}$ Inken Buengener ${ }^{1} \cdot$ Raphaela Grafiadeli $^{2}$ - Eva Heuthaler ${ }^{1}$. Judith Strieder ${ }^{1}$. \\ Patricia Ziehn $^{1} \cdot$ Christine Knaevelsrud $^{1} \cdot$ Babette Renneberg ${ }^{1} \cdot$ Johanna Boettcher $^{1,2}$
}

Accepted: 4 October 2021 / Published online: 31 October 2021

(c) The Author(s) 2021

\begin{abstract}
Background Transdiagnostic treatments target shared mechanisms between disorders to facilitate change across diagnoses. The Unified Protocol (UP) aims at changing dysfunctional reactions towards emotions by increasing mindful emotion awareness and cognitive flexibility, as well as decreasing anxiety sensitivity and emotion avoidance.

Method We investigated whether these transdiagnostic processes were malleable by treatment and mediated the relationship between treatment and outcome in an internet-delivered adaptation of the UP. $N=129$ participants with mixed anxiety, depressive, and somatic symptom disorders were randomized to treatment or waitlist.

Results The treatment yielded significant changes in all transdiagnostic processes over time in comparison to a waitlist condition. In separate mediator models, significant mediating effects were found for mindfulness, cognitive flexibility, behavioral activation, and experiential avoidance. When all mediators were combined in a multiple mediator model, the indirect effects through mindfulness and cognitive flexibility emerged as significant.

Conclusion These findings add to the growing body of research on transdiagnostic processes as mediators of change and emphasize mindfulness and cognitive flexibility as a transdiagnostic treatment target. However, these results should be interpreted cautiously, as temporal precedence could not be established.
\end{abstract}

Keywords Transdiagnostic $\cdot$ Internet-based cognitive behavioral therapy $\cdot$ Unified Protocol $\cdot$ Mediators $\cdot$ Mechanisms of change

\section{Introduction}

In recent years, the field of psychotherapy has seen a rise of novel transdiagnostic treatments that have the potential to provide easier access to evidence-based mental health care by addressing a wider range of disorders and lowering barriers to treatment (e.g., Dalgleish et al., 2020; SauerZavala, et al., 2017a, 2017b; Schaeuffele, et al., 2021b). Transdiagnostic treatments show promising effects when delivered face-to-face and over the internet (Newby et al., 2015; Păsărelu et al., 2017; Pearl \& Norton, 2017), with equivalence to gold-standard single disorder protocols

Carmen Schaeuffele

carmen.schaeuffele@fu-berlin.de

1 Department of Education and Psychology, Freie Universität Berlin, Berlin, Germany

2 Clinical Psychology and Psychotherapy, Psychologische Hochschule Berlin, Berlin, Germany (e.g., Barlow et al., 2017; Pearl \& Norton, 2017). However, overall - across single disorder and transdiagnostic treatments - there remains a need to improve effectiveness and response rates. A better understanding of the active ingredients of psychotherapy can help to create more effective treatments and lower non-response rates (Kazdin, 2007). With the emergence of personalized and modularized treatments, knowledge on change mechanisms can also help to create a better fit between therapy and patient (Hofmann \& Hayes, 2019). However, after years of research and advancements, the factors underlying change are still poorly understood (Cuijpers et al., 2019; Kazdin, 2007). This may be due to the fact that investigating mechanisms of change is a complicated endeavor. Determining whether a factor constitutes a (causal) mechanism of change requires a theoretical foundation of putative mechanisms to guide the investigation, the establishment of temporal precedence (change occurs first in the mediator followed by change in symptoms), the 
inclusion of several mediators, and ideally a manipulation of the mediating variable (Cuijpers et al., 2019; Kazdin, 2007).

A common distinction in mechanisms of change is between common and specific factors. Common factors are active across virtually all therapies and settings, whereas specific factors, as the name suggests, are more treatmentspecific (Cuijpers et al., 2019; Wampold, 2015). The most robust evidence on common factors has been found for therapeutic alliance and treatment expectancy (Baier et al., 2020; Constantino et al., 2018; Flückiger et al., 2018). Therapeutic alliance and treatment expectancy have also been shown to predict and mediate outcomes in the internet-based setting (Berger, 2017; Boettcher et al., 2013; El Alaoui et al., 2015, 2016; Flückiger et al., 2018; Probst et al., 2019). In comparison, the body of research on relevant specific factors is less clear (e.g., Domhardt et al., 2020; Kazantzis et al., 2018). The number of potential candidate mechanisms impedes the study of specific factors, with every disorderspecific model encompassing specific processes that cause or maintain the disorder. Evidence on how these specific processes are related to outcome is difficult to aggregate and summarize across disorders. In contrast, the transdiagnostic approach, with its emphasis on shared mechanisms that should be targeted in treatment, provides an attractive framework for investigating mediating variables (Sauer-Zavala, et al., 2017b).

The Unified Protocol (UP) is one example of a theorydriven transdiagnostic treatment for emotional disorders (Barlow et al., 2016; Ellard et al., 2010). The authors hypothesize that individuals with emotional disorders share a tendency to frequently and intensely experience negative affect and exhibit maladaptive reactions to these high negative emotions (Barlow et al., 2014; Bullis et al., 2019). The UP aims at changing these dysfunctional reactions towards emotions by increasing mindful emotion awareness as well as cognitive flexibility and decreasing avoidance (Barlow et al., 2004). These processes are increasingly recognized as transdiagnostic mediators of change across CBT treatments (e.g., Eustis et al., 2016; Goldin et al., 2016; Kocovski et al., 2015).

First studies started to investigate transdiagnostic processes in relation to outcome within the UP framework. As an important prerequisite, the modules of psychoeducation, mindful emotion awareness, cognitive flexibility, and countering emotional behaviors led to changes in these putative mechanisms (Sauer-Zavala, et al., 2017a). Single case experimental design studies found that mindfulness, cognitive flexibility, and anxiety sensitivity were associated with outcome (Boswell, 2013; Boswell et al., 2014; Brake et al., 2016). Findings on avoidance are mixed. In single case experimental design studies, experiential avoidance did not exhibit substantial changes following treatment (Boswell et al., 2014; Brake et al., 2016) and did not predict or proceed changes in anxiety or depression in an individual with comorbid depression and generalized anxiety (Boswell et al., 2014). In a larger RCT, Eustis et al. (2019) investigated the mediating effect of experiential avoidance across the UP and single-disorder treatment protocols for mixed anxiety disorders. In contrast to these single case studies, experiential avoidance mediated and preceded changes across the UP and single-disorder protocols, suggesting that experiential avoidance is a general transdiagnostic mechanism in CBT (Eustis et al., 2019). One meta-analysis aggregated the UP's effects on emotion regulation (Sakiris $\&$ Berle, 2019). They differentiated between adaptive (e.g., mindfulness, reappraisal) and maladaptive (e.g. experiential avoidance, thought suppression) emotion regulation skills (Sakiris \& Berle, 2019). Overall, they found preliminary evidence that adaptive strategies increased moderately over the course of treatment and that there were moderate to large effects on experiential avoidance and negligible effects on thought suppression.

Taken together, while there is preliminary evidence for the putative transdiagnostic mechanisms in the UP, the majority of findings are correlational, limited to anxiety disorders, and stem from small sample sizes. Only Eustis et al. (2019) investigated mediating effects in a larger sample, but their investigation was limited to one transdiagnostic process.

Our primary goal for this study was to extend previous findings on the relevance of transdiagnostic processes, specifically mindfulness, cognitive flexibility, anxiety sensitivity, and avoidance, in the UP in an internet-based setting for emotional disorders beyond anxiety disorders. We were interested whether these transdiagnostic processes were malleable by treatment and to which extent they mediated treatment effects - both in isolation and in a multiple mediator model taking into account their interrelation.

\section{Method}

We collected data within a randomized controlled trial examining the efficacy of an internet-based version of the UP for anxiety, depressive, and somatic symptom disorders (Schaeuffele et al., 2020). For this purpose, treatment was compared to waitlist in a 1:1 ratio $(N=129)$. The treatment yielded large effects on symptom distress in comparison to waitlist. $69 \%$ of participants in the treatment group were considered responders (as determined based on the reliable change index $(\mathrm{RCI}=1.96))$. The proportion of responders would shift to a more conservative estimate of $45 \%$ if we considered all participants that did not report post-treatment data as non-responders. Following the waiting period of 10 weeks, participants of the waitlist received access to the intervention. 


\section{Participants}

We recruited participants by self-selection in online mental health forums, on social media platforms, as well as over targeted ads on a popular search engine. We included participants with an anxiety, depressive, or somatic symptom disorder as a primary diagnosis in the trial. While the UP has primarily been applied in the treatment of anxiety disorders (e.g., Cassiello-Robbins et al., 2020), the rationale should be expandable to other disorders with strong emotional components like depression and somatic symptom disorders (Farchione et al., 2012). Participants were diagnosed with a structured clinical interview based on DSM-5 via telephone prior to randomization (Diagnostic interview for mental disorders (DIPS); Margraf et al., 2017). We excluded participants if they (1) currently underwent psychotherapy, (3) were under 18 , (4) changed their medication dosage in the preceding three months, (5) showed indications of acute suicidality, (6) were diagnosed with a substance use disorder, (7) experienced symptoms of mania, or (8) psychosis. See Fig. 1 for a detailed flow of participants through the inclusion process.

\section{Intervention}

The intervention is a 10-week adaptation of the UP for internet-based use. For this purpose, the UP workbook was translated one-to-one from English into German. In a next step, the content was divided into ten modules. In several editing loops, the paragraphs were shortened, simplified, and summarized, while retaining the core content and exercises. Figure 2 shows core concepts of the 10 final modules. Participants had to complete a module in order to start with a new module. To complete a module, participants had to complete a reflection on how this module helped them work towards their goals. We recommended that participants work on one module per week, but participants were free to complete the modules at their own pace. In theory, participants could skip exercises and leave the reflection blank to start a new module, but this only affected a negligible proportion of modules and participants. Participants on average completed 7.22 modules. $73 \%$ of participants completed the modules corresponding to the core treatment targets of the UP (mindfulness, cognitive flexibility, avoidance) and $42 \%$ completed all ten modules. Online therapists were the first and last author as well as 16 advanced students of clinical psychology who were trained in a 1-day workshop and supervised. Online therapists provided written feedback once per week on a fixed day over the course of 10 weeks. Therapists based their feedback on the record sheets that participants completed within the online therapy program. The therapists focused their written feedback on increasing engagement and motivation as well as providing guidance on how to work and advance with the therapeutic materials. Online therapists, for example, provided guidance when they felt that participants did not engage with the exercises in the intended manner or struggled with adaptive solutions (e.g. therapists would guide participants to come up with alternative adaptive behaviors or offer personalized examples of possible alternative thoughts).

\section{Ethics and Trial Registration}

The ethics committee of the Department of Education and Psychology at Freie Universitaet Berlin, Germany, approved the trial (186/2018). The trial was registered at the German Clinical Trial Registry DRKS (DRKS00014820).

\section{Measures}

All assessments were self-rated on the online platform. Figure 2 shows an overview of measurement time points. Outcome and mediators were assessed at baseline $\left(\mathrm{t}_{0}\right)$. All putative mediators were assessed immediately following the module that targeted the construct to ensure participants had received the dosage of treatment $\left(t_{1}\right)$. For mechanisms targeted early in treatment (mindfulness and cognitive flexibility), we additionally administered the measures at posttreatment to explore further changes $\left(\mathrm{t}_{2}\right)$. We did not assess anxiety sensitivity and avoidance at post-treatment due to temporal proximity to the module 9/week 9 assessment. We assumed that one module was completed per week. Accordingly, we assessed the transdiagnostic processes in the waitlist group in the week that corresponded to the number of the module (e.g., we assessed mindfulness after module 3 in the treatment and after 3 weeks in the waitlist group). The percentage of missing values was comparatively high (see Table 2). Technical problems at the beginning of the study led to low response rates in the waitlist control group. As many participants in the intervention group did not complete all modules, missingness rates were higher for measures administered in later modules.

\section{Symptom Distress}

We assessed symptom distress with the 18-item Brief Symptom Inventory (BSI-18) (Derogatis, 2000; German version: Franke et al., 2011). The BSI-18 is internally reliable and demonstrates satisfactory psychometric properties (Derogatis \& Fitzpatrick, 2004; Franke et al., 2011; Prinz et al., 2013).

\section{Mindfulness}

We assessed mindfulness with the 16-item Southampton Mindfulness Questionnaire (SMQ) (Chadwick 


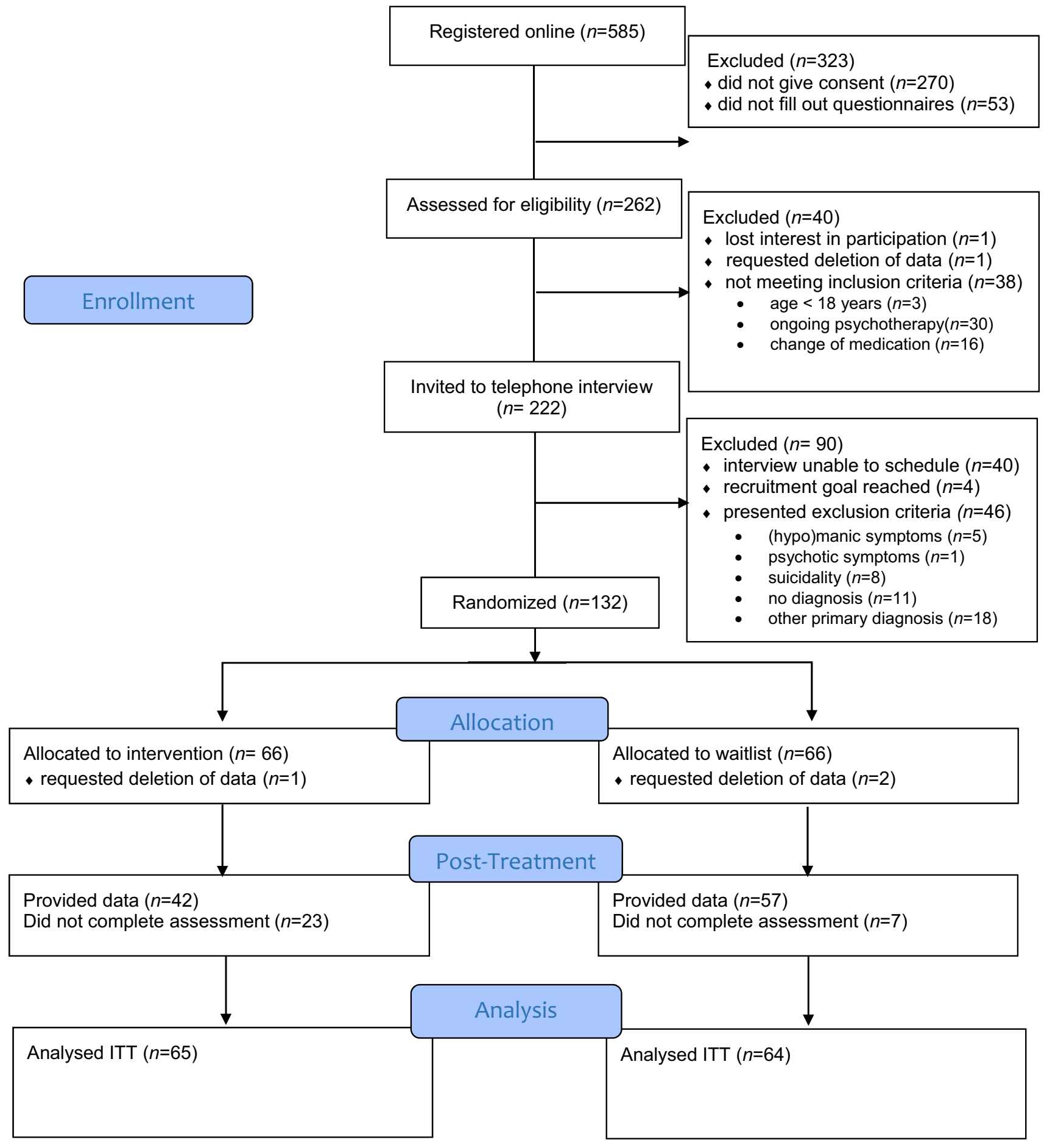

Fig. 1 Consort flow chart

et al., 2008; German version: Böge et al., 2020). The SMQ assesses mindfulness as the reaction to distressing thoughts and images. Example items include "Usually when I experience distressing thoughts and images, I judge the thought/image as good or bad" and "Usually when I experience distressing thoughts and images, I just notice them and let them go". The SMQ is unidimensional and internally reliable (Böge et al., 2020; Chadwick et al., 2008). 


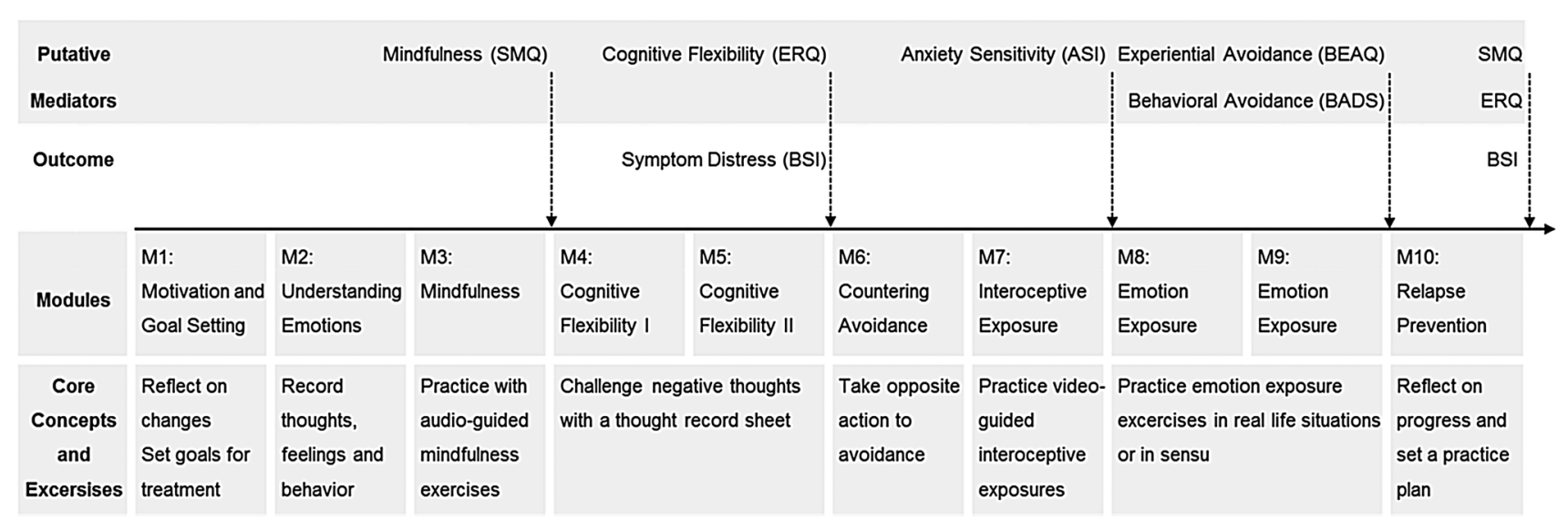

Fig. 2 Core concepts of the intervention and measurement points of mediators and outcome. SMQ Southampton Mindfulness Questionnaire, BSI Brief Symptom Distress Inventory, ERQ Emotion Regula-

\section{Cognitive Flexibility}

We assessed cognitive flexibility with the 6-item reappraisal subscale of the Emotion Regulation Questionnaire (ERQ) (Gross \& John, 2003; German version: Abler \& Kessler, 2009). Example items include "When I want to feel less negative emotion, I change the way I' $m$ thinking about the situation." and "I control my emotions by changing the way I think about the situation I'm in". The ERQ has satisfactory psychometric properties (Abler \& Kessler, 2009; Gross \& John, 2003).

\section{Anxiety Sensitivity}

We assessed anxiety sensitivity with the 18-item Anxiety Sensitivity Index-3 (ASI-3) (Taylor et al., 2007; German version: Kemper et al., 2009). The scale assesses three facets of anxiety sensitivity: Physical, cognitive, and social concerns. Respective example items include "When my chest feels tight, I get scared that I won't be able to breathe properly", "When my mind goes blank, I worry there is something terribly wrong with me.", and "When I begin to sweat in a social situation, I fear people will think negatively of me.". Psychometric properties are satisfactory (Kemper et al., 2009; Taylor et al., 2007).

\section{Avoidance}

The UP targets avoidance by countering avoidance tendencies and engaging in emotional exposures. For depressed patients specifically, targeting avoidance relates to overcoming tendencies of withdrawal and engaging more actively with valued activities which in turn should increase activation (Boswell et al., 2018). In order to capture different aspects of avoidance and be more reflective of the proportion tion Questionnaire, $A S I$ Anxiety Sensitivity Index, $B E A Q$ Brief Experiential Avoidance Questionnaire, BADS Behavioral Activation for Depression Scale

of depressed participants in our sample, we assessed both experiential avoidance, which has been described as the unwillingness to remain in contact with distressing emotions, thoughts, memories, and physical sensations, as well as behavioral activation.

Experiential Avoidance We assessed experiential avoidance with the 15-item Brief Experiential Avoidance Questionnaire (BEAQ) (Gámez et al., 2014; German version: Schaeuffele, et al., 2021a). The BEAQ is internally reliable (Gámez et al., 2014). Example items include "The key to a good life is never feeling any pain" and "I go out of my way to avoid uncomfortable situations". We used our own German translation of the scale. Psychometric properties were investigated in a student and clinical population with comparable results to the English original. Our translation showed high internal reliability (.80-.81), 7 to 13-day testretest-reliability (.77-.86), and a favorable pattern of convergent and divergent validity in comparison to other measures of experiential avoidance. Our findings indicated that the BEAQ is well represented by a bifactor structure that includes a general experiential avoidance factor and specific factors of individual facets of experiential avoidance (e.g., distress aversion or distraction). Future studies should substantiate this preliminary finding. For the purpose of this study, we relied on the total scale.

Behavioral Activation We assessed behavioral avoidance and activation with the 9-item Behavioral Activation for Depression Scale Short Form (BADS) (Manos et al., 2011; German version: Fuhr et al., 2016). The BADS has two subscales, an activation and an avoidance subscale. While originally developed to measure changes during behavioral activation for depression, items are phrased transdiagnostically. Example items include "I engaged in a wide and diverse array of 
activities" or "Most of what I did was to escape from or avoid something unpleasant". The instrument has shown good psychometric properties (Fuhr et al., 2016; Manos et al., 2011).

\section{Statistical Analyses}

The accompanying de-identified data set is available at https:// osf.io/hr5wb/. We ran all statistical analyses in RStudio, version 1.2.5033 (RStudio Team, 2019). For the transdiagnostic processes, we first investigated whether the treatment led to significant changes in the putative mediators (treatment, time, and the interaction of treatment and time as fixed and individuals as random effects, mediator as outcome) with linear mixed effects models using the lme4 package (Bates et al., 2014), obtaining $\mathrm{p}$ values with the lmer-test package (Kuznetsova et al., 2017). We also calculated effect sizes (Hedge's $g$ ) using estimated means and pooled standard deviations.

We then tested all putative transdiagnostic processes in separate mediator models with group as predictor and symptom distress at post-treatment as outcome (see Fig. 3). For mindfulness and cognitive flexibility two time points were included in these models, while for anxiety sensitivity, avoidance, and activation only one time point was available for the comparison. In a next step, we combined all mediators that showed a significant mediating effect in the separate mediator models in a multiple mediator model (see Fig. 4), where residuals of mediators were allowed to covary (Preacher \& Hayes, 2008). Again, two time points were included for mindfulness and cognitive flexibility. Mediation models were fitted as path analyses with bias-corrected bootstrapping (10,000 draws) and full maximum likelihood estimation to account for missing values using the lavaan package (Rosseel, 2012). If the bias-corrected bootstrapped confidence interval does not contain zero, then the effect of treatment is at least partially dependent on the mediator and the indirect effect can be considered significant (Preacher \& Hayes, 2008). We controlled all mediators and outcomes for their baseline value, following findings from a simulation study that found this approach superior to other methods like difference scores in terms of error rates, bias, power, and confidence interval coverage (Valente \& MacKinnon, 2017). To judge the magnitude of the mediating effect, $P_{M}$ - the ratio of the indirect effect to the total effect-was calculated (Preacher \& Kelley, 2011; Wen \& Fan, 2015).

\section{Results}

\section{Demographics}

Demographic variables are displayed in Table 1. Groups did not differ at baseline.

\section{Transdiagnostic Processes over Time}

Means, Hedges' $g$ effect sizes, as well as percentages of missing values at each measurement point are reported in Table 2. We found significant changes over time in comparison to waitlist for all transdiagnostic processes: mindfulness, cognitive flexibility, anxiety sensitivity, behavioral activation, and experiential avoidance $(F=5.30-16.40, d f=1-2$, $p<0.05)$. The transdiagnostic processes changed in the hypothesized direction: mindfulness, cognitive flexibility, and behavioral activation increased $(g=0.73-0.99)$, while anxiety sensitivity and experiential avoidance decreased $(g=0.4-0.48)$. Between group effect sizes were large for behavioral activation and cognitive flexibility $(g=0.86$ 0.88 ), and medium for mindfulness, anxiety sensitivity, and experiential avoidance $(g=0.59-0.76)$.

\section{Transdiagnostic Processes as Mediators}

Unstandardized and standardized parameter estimates as well as fit indices of the full models can be found in the supplementary material.

\section{Single Mediator Models}

Single mediator models with transdiagnostic processes as the mediator between group and outcome are depicted in Fig. 3. For mindfulness and cognitive flexibility, two measurement points were available for the comparison between treatment and waitlist and the indirect effects through both time points were significant, as shown in Fig. 3. For mindfulness, the indirect effect through the first time point was $0.93,90 \%$ CI $[0.18,2.19]$ and for the second $-2.57,90 \% \mathrm{CI}$ [-4.86, -0.67]. For cognitive flexibility, the indirect effect through the first time point was $-0.59,90 \% \mathrm{CI}[-1.71$, $0.33]$ and for the second $-1.06,90 \%$ CI $[-2.74,-0.06]$. The indirect effect through anxiety sensitivity was not significant, while the indirect path through experiential avoidance and behavioral activation were. The treatment effect, thus, was partially mediated by mindfulness, cognitive flexibility, experiential avoidance, and behavioral activation, but not anxiety sensitivity. The effect size of the mediating effect was $P_{M}=.13$ for mindfulness (indirect effect through $\mathrm{t} 1$ and t2), $P_{M}=.06$ for cognitive flexibility (indirect effect through $\mathrm{t} 1$ and $\mathrm{t} 2), P_{M}=.09$ for experiential avoidance, and $P_{M}=.15$ for behavioral avoidance.

\section{Multiple Mediator Models}

Figure 4 depicts the multiple mediator model that includes all significant mediators from the single mediator models (all except anxiety sensitivity). In the combined multiple mediator model, the bootstrapped $95 \%$ confidence interval 
Fig. 3 Single mediator models: indirect effect of intervention on symptom distress through transdiagnostic processes. Note. Graphical depiction of the mediator models of (1) mindfulness, (2) cognitive flexibility, (3) anxiety sensitivity, (4) experiential avoidance, and (5) behavioral activation as mediators between intervention and symptom distress. Mindfulness and cognitive flexibility were measured twice, after the module that targeted the mechanism (module/week 3 and module/week 5 respectively;

(t1) and at post-treatment (t2). Anxiety sensitivity, experiential avoidance, and behavioral activation were solely assessed after the module that targeted them (module/week 7 or module/week 9). Symptom distress was measured at post-treatment. Mediators and outcome were controlled by their respective baseline value. These paths were omitted from the figure for clarity. CIs represent upper and lower limit of 95\% Bootstrapcorrected confidence intervals. $* p<.05$
(1) Mediation through Mindfulness

indirect effect through t1 and t2: -1.2 [-2.39, -0.4]

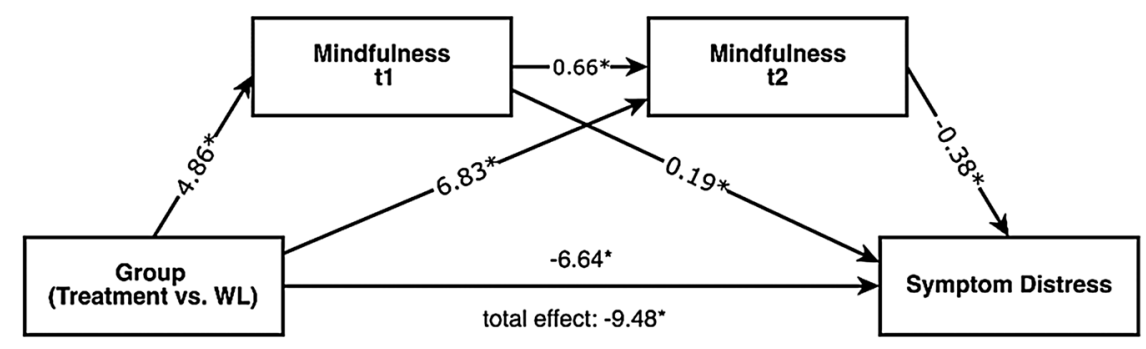

(2) Mediation through Cognitive Flexibility

indirect effect through $t 1$ and t2: $-0.61[-1.93,-0.03]$

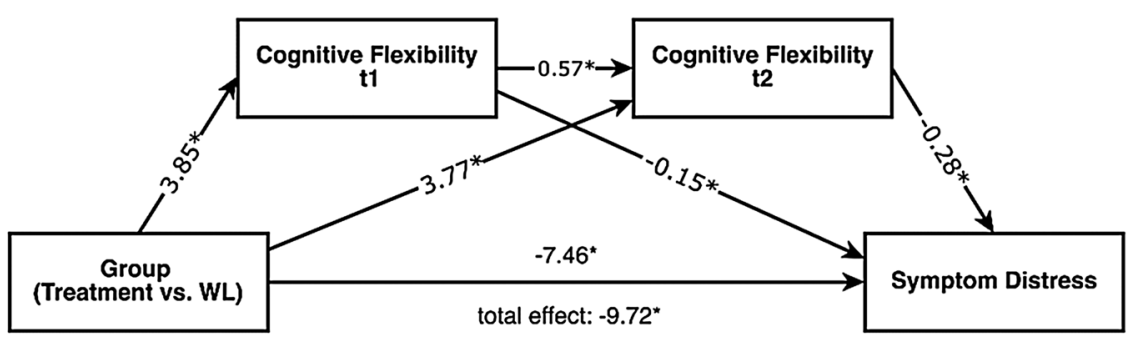

(3) Mediation through Anxiety Sensitivty

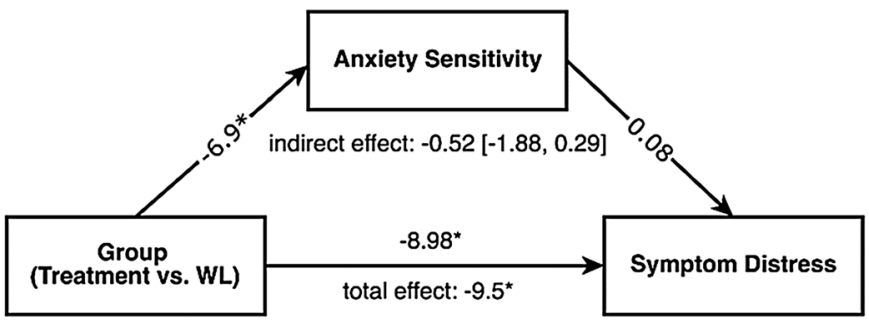

(4) Mediation through Experiential Avoidance

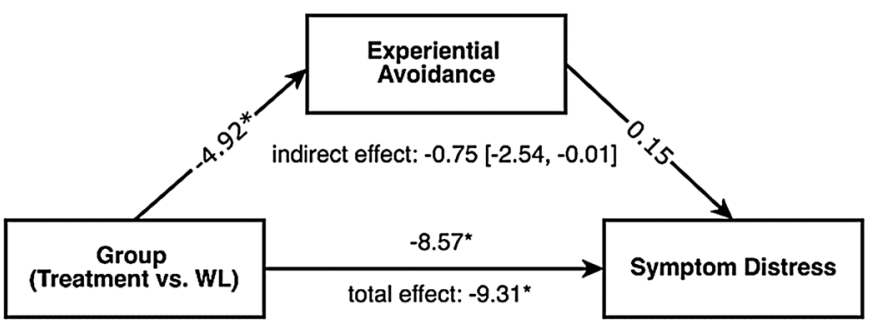

(5) Mediation through Behavioral Activation

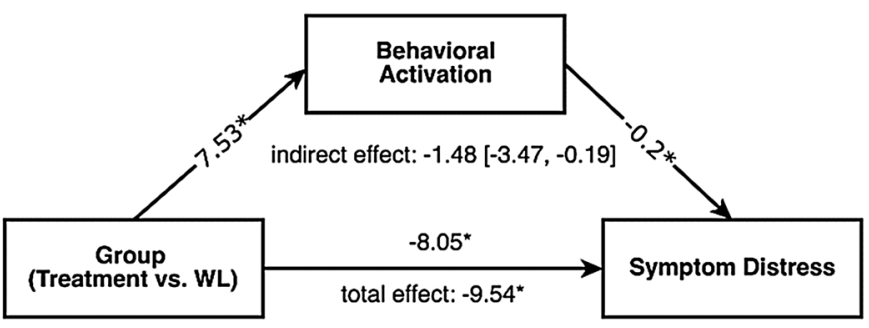




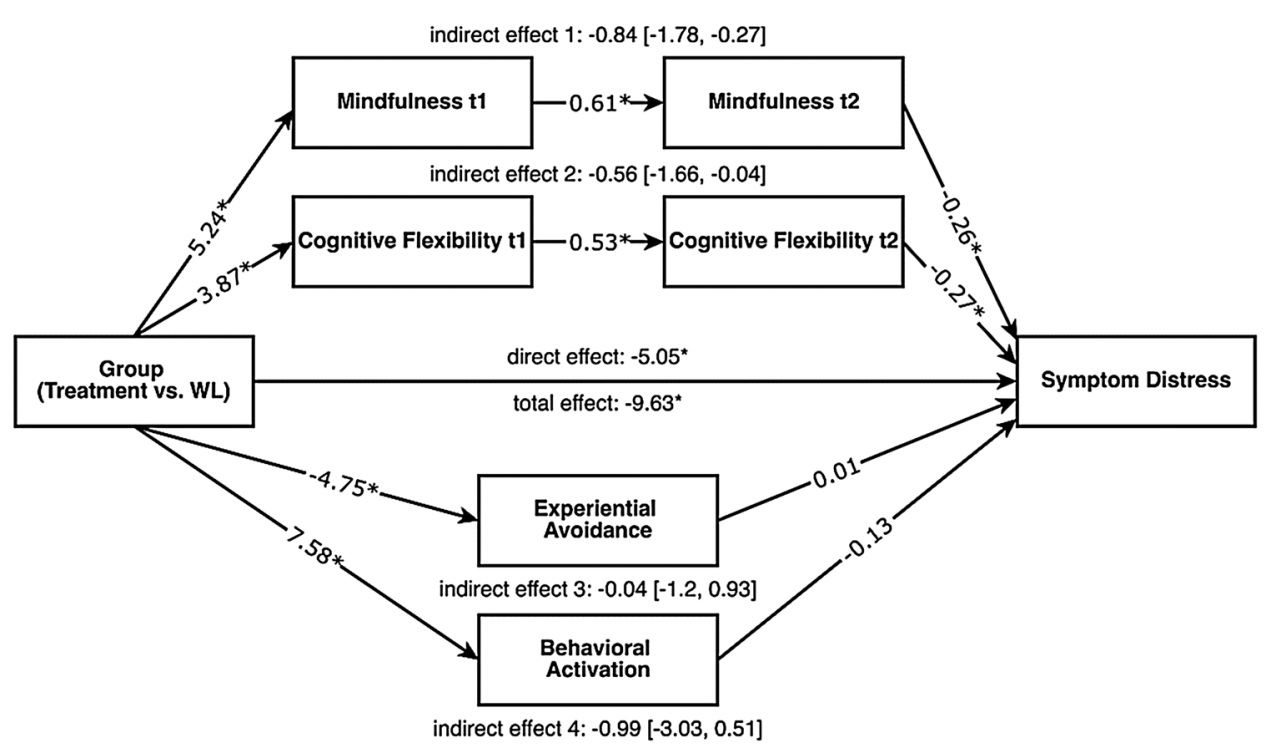

Fig. 4 Multiple mediator model. Note. Graphical depiction of the multiple mediation model including mindfulness, cognitive flexibility, experiential avoidance, and behavioral activation as mediators between intervention and symptom distress. Mindfulness and cognitive flexibility were measured twice, after the module that targeted the mechanism (module/week 3 and module/week 5 respectively; $t$ 1) and at post-treatment (t2). Experiential avoidance and behavioral acti-

of the indirect path through mindfulness and cognitive flexibility (through both time points) did not contain zero: The treatment effect was partially mediated by mindfulness and cognitive flexibility, but not by the other putative processes. The indirect effect through mindfulness showed an effect size of $P_{M}=.09$ and through cognitive flexibility $P_{M}=.06$. The total indirect effect (all indirect effects combined) was -4.58 , corresponding to an effect size of $P_{M}=.48$.

\section{Discussion}

This study investigated transdiagnostic processes in an internet-based intervention based on the Unified Protocol for anxiety, depressive, and somatic symptom disorders. All transdiagnostic processes were malleable by treatment with medium to large effect sizes. In single mediator models, we found that cognitive flexibility, mindfulness, experiential avoidance, and behavioral activation partially mediated the relationship between treatment and symptom distress. When all significant mediators were collapsed into one model, the multiple mediation model revealed the indirect paths through mindfulness and cognitive flexibility as significant.

Results from the single mediator models are in line with previous research on the UP that found mindfulness, cognitive flexibility, and experiential avoidance predictive and associated with symptom change (Boswell et al., 2014; Brake et al., 2016; Eustis et al., 2019). Anxiety vation were solely assessed after module/week 9. Symptom distress was measured at post-treatment. Mediators and outcome were controlled by their respective baseline value and mediators were allowed to covary. These paths were omitted from the figure for clarity. Indirect effect 1 and 2 refer to the indirect effects through t 1 and t2. CIs represent upper and lower limit of 95\% Bootstrap-corrected confidence intervals. $* p<.05$

sensitivity - while increasingly conceptualized as a transdiagnostic process and found to be associated with symptom change in the UP (Boettcher et al., 2016; Boswell et al., 2013) - might play a lesser role in depressive disorders and thus, may not be related to outcome to the same extent in our fairly depressed sample. Future studies should investigate whether these findings on transdiagnostic processes are dependent on diagnosis, as suggested by a recent study on mindfulness as a predictor of outcome in the UP (Woods et al., 2020). The non-significant finding on anxiety sensitivity also raises the question of whether all participants benefit from the interoceptive exposure module that targets anxiety sensitivity. This highlights the potential of a flexible, modular application of the UP, where an individualized sequence of treatment components could be provided based on transdiagnostic processes (Fisher et al., 2019; Sauer-Zavala et al., 2018).

To our knowledge, transdiagnostic processes have not been investigated jointly in one mediation model for the UP as of yet. Mindfulness and cognitive flexibility, as measured by the SMQ and the reappraisal subscale of the ERQ, might capture the overarching aim of the UP best with their focus on how individuals react to distressing thoughts and images (and by that probably also emotions). These findings also tap into the debate whether it is more beneficial to decrease maladaptive or increase adaptive emotion regulation strategies in treatment (e.g., Southward et al., 2021). In our study, the adaptive emotion 
Table 1 Demographic characteristics of participants at baseline

\begin{tabular}{|c|c|c|c|c|c|c|}
\hline \multirow[t]{2}{*}{ Demographic variable } & \multirow[t]{2}{*}{ Treatment $(n=65)$} & \multirow[t]{2}{*}{ Waitlist $(n=64)$} & \multirow[t]{2}{*}{ Total } & \multicolumn{3}{|c|}{$\begin{array}{l}\text { Statistical test of } \\
\text { group difference }\end{array}$} \\
\hline & & & & df & $\chi^{2 / t}$ & $\mathrm{p}$ \\
\hline Gender & & & & 1 & .48 & .49 \\
\hline Male & $23(35.4 \%)$ & $18(28.1 \%)$ & $41(31.8 \%)$ & & & \\
\hline Female & $42(64.6 \%)$ & $46(71.9 \%)$ & $88(68.2 \%)$ & & & \\
\hline Age & & & & 127 & .18 & .86 \\
\hline Mean (SD) & $37.51(11.99)$ & $37.11(13.04)$ & $37.31(12.47)$ & & & \\
\hline Range & $18-67$ & $18-66$ & $18-67$ & & & \\
\hline Relationship & & & & 1 & 1.99 & .16 \\
\hline In a relationship & $40(61.5 \%)$ & $30(46.9 \%)$ & $70(54.3 \%)$ & & & \\
\hline Single & $25(38.5 \%)$ & $34(53.1 \%)$ & $59(45.7 \%)$ & & & \\
\hline Highest education & & & & 4 & 4.7 & .32 \\
\hline Up to 9 years of school education & $4(6.2 \%)$ & $5(7.8 \%)$ & $9(7 \%)$ & & & \\
\hline Secondary school & $10(15.4 \%)$ & $11(17.2 \%)$ & $21(16.3 \%)$ & & & \\
\hline College entrance qualification & $16(24.6 \%)$ & $25(39.1 \%)$ & $41(31.8 \%)$ & & & \\
\hline College/university degree & $34(52.3 \%)$ & $22(34.4 \%)$ & $56(43.3 \%)$ & & & \\
\hline Other & $1(1.5 \%)$ & $1(1.6 \%)$ & $2(1.6 \%)$ & & & \\
\hline Employment status & & & & 5 & 2.99 & .7 \\
\hline Employed & $33(50.8 \%)$ & $29(45.3 \%)$ & $62(48.1 \%)$ & & & \\
\hline Self-employed & $4(6.2 \%)$ & $5(7.8 \%)$ & $9(7 \%)$ & & & \\
\hline In education & $12(18.5 \%)$ & $17(26.6 \%)$ & $29(22.5 \%)$ & & & \\
\hline Pensioned & $3(4.6 \%)$ & $5(7.8 \%)$ & $8(6.2 \%)$ & & & \\
\hline Unemployed & $9(13.8 \%)$ & $6(9.4 \%)$ & $15(11.6 \%)$ & & & \\
\hline Other & $4(6.2 \%)$ & $2(3.1 \%)$ & $6(4.7 \%)$ & & & \\
\hline Currently on medication & & & & 1 & 0 & .99 \\
\hline Yes & $16(24.6 \%)$ & $16(25 \%)$ & $32(24.8 \%)$ & & & \\
\hline No & $49(75.4 \%)$ & $48(75 \%)$ & $97(75.2 \%)$ & & & \\
\hline Prior psychotherapy & & & & 1 & 1.95 & .16 \\
\hline Yes & $44(67.7 \%)$ & $51(79.7 \%)$ & $95(73.6 \%)$ & & & \\
\hline No & $21(32.3 \%)$ & $13(20.3 \%)$ & $34(26.4 \%)$ & & & \\
\hline Primary diagnosis & & & & 9 & 5.66 & .77 \\
\hline Agoraphobia & $3(4.6 \%)$ & $4(6.3 \%)$ & $7(5.4 \%)$ & & & \\
\hline Generalized anxiety disorder & $9(13.8 \%)$ & $5(7.8 \%)$ & $14(10.8 \%)$ & & & \\
\hline Panic disorder & $5(7.7 \%)$ & $5(7.8 \%)$ & $10(7.8 \%)$ & & & \\
\hline Social anxiety disorder & $13(20 \%)$ & $16(25 \%)$ & $29(22.5 \%)$ & & & \\
\hline Major depressive disorder & $14(21.5 \%)$ & $17(26.6 \%)$ & $31(24 \%)$ & & & \\
\hline Persistent depressive disorder & $15(23.1 \%)$ & $12(18.8 \%)$ & $27(20.9 \%)$ & & & \\
\hline Somatic symptom disorder & $2(3.1 \%)$ & $3(6.3 \%)$ & $5(3.9 \%)$ & & & \\
\hline Illness anxiety disorder & $4(6.2 \%)$ & $2(3.1 \%)$ & $6(4.7 \%)$ & & & \\
\hline \multicolumn{7}{|l|}{ Comorbidity } \\
\hline 1 Comorbid diagnosis & $51(78.5 \%)$ & $48(75 \%)$ & $99(76.7 \%)$ & & & \\
\hline 2 Comorbid diagnoses & $27(41.5 \%)$ & $28(43.7 \%)$ & $55(42.6 \%)$ & & & \\
\hline 3 Comorbid diagnoses & $18(27.7 \%)$ & $18(28.1 \%)$ & $36(27.9 \%)$ & & & \\
\hline 4 Comorbid diagnoses & $12(18.5 \%)$ & $10(15.6 \%)$ & $22(17.1 \%)$ & & & \\
\hline 5 Comorbid diagnoses & $5(7.7 \%)$ & $4(6.2 \%)$ & $9(6.9 \%)$ & & & \\
\hline
\end{tabular}

regulation processes - mindfulness, cognitive flexibility, and behavioral activation - seemed to show more stable indirect effects than experiential avoidance (and anxiety sensitivity).
The disentanglement of transdiagnostic processes seems quite complex, given they are all related, overlapping, and may share a common underlying basis (e.g., Mansell \& McEvoy, 2017; Spinhoven et al., 2017). For instance, 


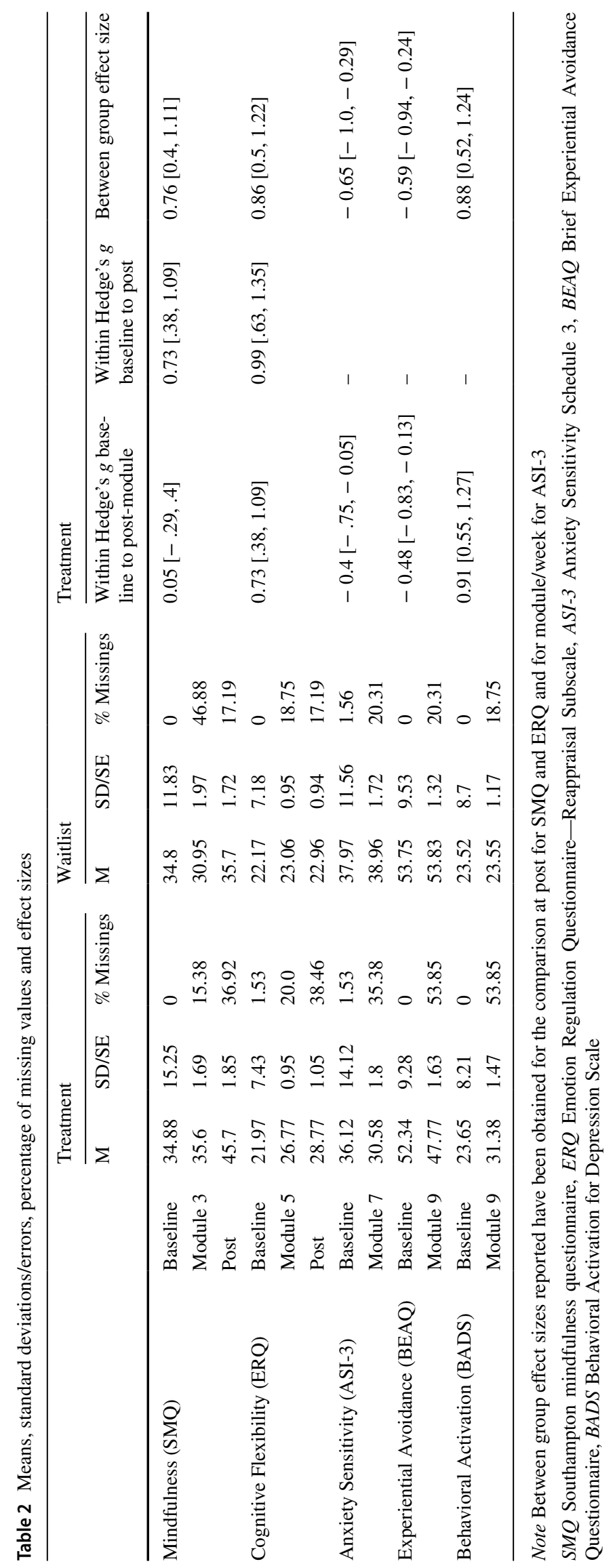


mindfulness training seems to facilitate exposure and decrease avoidance in some participants treated with the UP, highlighting the interplay and carryover effects between processes (Curreri et al., 2020). CBT treatments have also shown to be mediated by transdiagnostic processes without explicitly targeting them, for example by mindfulness (e.g., Goldin et al., 2016; Kocovski et al., 2015) or experiential avoidance (e.g., Eustis et al., 2016). Thus, based on our results, we cannot conclude that other treatment elements, besides mindfulness training and cognitive restructuring, are obsolete, since modules targeting avoidance may also enhance mindful emotion awareness and cognitive flexibility. These findings also challenge our theory-driven decision to measure mechanisms following the module that target them. Instead, additional research on the structure and hierarchy of transdiagnostic processes as well as dismantling component studies are needed to get a better understanding of the interplay of the processes in therapy and isolate effects.

Our findings need to be interpreted in the light of several limitations. First, the trial suffered from a high rate of missing values. Besides participants in the treatment group failing to fill out post-treatment assessments, dropout from the intervention affected the assessment of the transdiagnostic processes in the later modules. In order to ensure that participants received the proper dosage of treatment, we measured the transdiagnostic processes following the module that targeted them. But since the treatment was self-paced and the mean number of modules completed was $M=7.22$ (Schaeuffele et al., 2020), this naturally meant that the assessments after completion of module 7 and 9 were limited to participants who progressed to this stage of treatment. Thus, our findings are preliminary and should be replicated with larger samples. Our sample size also did not permit us to analyze mediation in different subsamples, e.g. responders versus non-responders. However, we believe that examining mechanisms of change particularly in those patients experiencing change is a promising route of future research (DeRubeis et al., 2014). The dropout also limits the generalizability of our results. We have several hypotheses as to why participants might have not completed the intervention. The self-paced setup and "one size fits all" transdiagnostic approach of the intervention may have been overwhelming and lacked personalization for participants, especially in a guided self-help context. In addition, the lack of adherence-fostering features like personalized reminders and progress reinforcement, as they are implemented in other guided internet-based interventions, may have been detrimental to adherence. Future applications could address these concerns by delivering the intervention in a personalized, modular fashion and including adherence-fostering features. Another limitation concerns the lack of temporal precedence that has been described as an important prerequisite for causation (Kazdin, 2007). When comparing treatment and waitlist, temporal precedence could not be established with how measurements were set up. While several other of the quality criteria of mediation studies outlined by Lemmens et al. (2016) could be established (RCT with control group, $n>40$ per group, assessment of several mediators), the lack of temporal precedence does not allow to draw any conclusions on causation. However, internet-based treatments are shorter in comparison to face-to-face and it seems to take time for the effect on transdiagnostic processes to unfold as the delayed changes in mindfulness and cognitive flexibility indicate. These circumstances complicate the establishment of temporality. Our results were obtained within an internet-based delivery of the UP. While the face-to-face and internetbased setting generally seem to share findings on relevant mechanisms of change (e.g., for depression: Domhardt et al., 2020; Lemmens et al., 2016), we cannot rule out that setting-specific influences affected our findings and they, thus, may not translate to the face-to-face setting to the full extent. Direct comparisons between face-toface and internet-based deliveries and their accompanying change mechanisms are sparse and should be subject of future studies. Linear mediation models, as employed in our study, may not be suitable to capture the complexity of therapeutic processes, since the relationship between outcome and mediator are dynamic, bidirectional, and non-linear (Hofmann et al., 2020). More frequent and parallel assessments of mediators and outcomes are needed to understand the dynamic and agents of change in a more fine-grained manner. To facilitate this, shorter and change sensitive measures should be utilized.

Identifying the active ingredient of treatments and moving towards personalized treatments might benefit response rates. This also applies to our study where nonresponse rates of $31 \%$ (or $55 \%$ as a more conservative estimate) - while in line with meta-analytic findings on response rates in internet-based settings (Rozental et al., 2019) - may be improved by providing more effective and personalized treatments. How could this be achieved? Our findings suggest that (internet-based) applications of the UP should especially emphasize and increase a mindful stance towards emotions as well as regulating emotions by cognitive reappraisal in order to enhance effects. Future studies should substantiate our findings with larger sample sizes and more frequent and parallel assessments.

Supplementary Information The online version contains supplementary material available at https://doi.org/10.1007/s10608-021-10272-y .

Funding Open Access funding enabled and organized by Projekt DEAL. This research did not receive any specific grant from funding agencies in the public, commercial, or not-for-profit sectors. 


\section{Declarations}

Conflict of interest Carmen Schaeuffele, Jonathan Bär, Inken Buengener, Raphaela Grafiadeli, Eva Heuthaler, Judith Strieder, Patricia Ziehn, Christine Knaevelsrud, Babette Renneberg, and Johanna Boettcher do not have any conflict of interest to report.

Informed Consent All participants completed the informed consent process before participating in the present research study.

Research Involving Human and Animal Participants No animal studies were carried out by the authors for this article.

Open Access This article is licensed under a Creative Commons Attribution 4.0 International License, which permits use, sharing, adaptation, distribution and reproduction in any medium or format, as long as you give appropriate credit to the original author(s) and the source, provide a link to the Creative Commons licence, and indicate if changes were made. The images or other third party material in this article are included in the article's Creative Commons licence, unless indicated otherwise in a credit line to the material. If material is not included in the article's Creative Commons licence and your intended use is not permitted by statutory regulation or exceeds the permitted use, you will need to obtain permission directly from the copyright holder. To view a copy of this licence, visit http://creativecommons.org/licenses/by/4.0/.

\section{References}

Abler, B., \& Kessler, H. (2009). Emotion Regulation QuestionnaireEine deutschsprachige Fassung des ERQ von Gross und John. Diagnostica, 55(3), 144-152. https://doi.org/10.1026/0012-1924. 55.3.144

Baier, A. L., Kline, A. C., \& Feeny, N. C. (2020). Therapeutic alliance as a mediator of change: A systematic review and evaluation of research. Clinical Psychology Review, 82, 101921. https://doi.org/ 10.1016/j.cpr.2020.101921

Barlow, D. H., Allen, L. B., \& Choate, M. L. (2004). Toward a unified treatment for emotional disorders. Behavior Therapy, 35(2), 205-230. https://doi.org/10.1016/S0005-7894(04)80036-4

Barlow, D. H., Allen, L. B., \& Choate, M. L. (2016). Toward a unified treatment for emotional disorders: Republished article. Behavior Therapy, 47(6), 838-853. https://doi.org/10.1016/j.beth.2016.11. 005

Barlow, D. H., Ellard, K. K., Sauer-Zavala, S., Bullis, J. R., \& Carl, J. R. (2014). The origins of neuroticism. Perspectives on Psychological Science, 9(5), 481-496. https://doi.org/10.1177/17456 91614544528

Barlow, D. H., Farchione, T. J., Bullis, J. R., Gallagher, M. W., MurrayLatin, H., Sauer-Zavala, S., Bentley, K. H., Thompson-Hollands, J., Conklin, L. R., Boswell, J. F., Ametaj, A., Carl, J. R., Boettcher, H. T., \& Cassiello-Robbins, C. (2017). The Unified Protocol for transdiagnostic treatment of emotional disorders compared with diagnosis-specific protocols for anxiety disorders: A randomized clinical trial. JAMA Psychiatry. https://doi.org/10.1001/jamap sychiatry.2017.2164

Bates, D., Mächler, M., Bolker, B., \& Walker, S. (2014). Fitting linear mixed-effects models using lme4. [Stat]. http://arxiv.org/abs/ 1406.5823

Berger, T. (2017). The therapeutic alliance in internet interventions: A narrative review and suggestions for future research.
Psychotherapy Research, 27(5), 511-524. https://doi.org/10.1080/ 10503307.2015.1119908

Boettcher, H., Brake, C. A., \& Barlow, D. H. (2016). Origins and outlook of interoceptive exposure. Journal of Behavior Therapy and Experimental Psychiatry, 53, 41-51. https://doi.org/10.1016/j. jbtep.2015.10.009

Boettcher, J., Renneberg, B., \& Berger, T. (2013). Patient expectations in internet-based self-help for social anxiety. Cognitive Behaviour Therapy, 42(3), 203-214. https://doi.org/10.1080/16506073.2012. 759615

Böge, K., Schaeuffele, C., Jacobsen, P., Chadwick, P., Ergen, E., Hahne, I., Bergmann, N., Boettcher, J., Wingenfeld, K., Bajbouj, M., \& Hahn, E. (2020). Validation of the German version of the Southampton Mindfulness Questionnaire (SMQ). Mindfulness, 11(9), 2219-2234. https://doi.org/10.1007/s12671-020-01447-x

Boswell, J. F. (2013). Intervention strategies and clinical process in transdiagnostic cognitive-behavioral therapy. Psychotherapy (chicago, Ill.), 50(3), 381-386. https://doi.org/10.1037/a0032157

Boswell, J. F., Anderson, L. M., \& Barlow, D. H. (2014). An idiographic analysis of change processes in the unified transdiagnostic treatment of depression. Journal of Consulting and Clinical Psychology, 82(6), 1060-1071. https://doi.org/10.1037/ a0037403

Boswell, J. F., Conklin, L. R., Oswald, J. M., \& Bugatti, M. (2018). The unified protocol for major depressive disorder. In Applications of the Unified Protocol for Transdiagnostic Treatment of Emotional Disorders. Oxford University Press.

Boswell, J. F., Farchione, T. J., Sauer-Zavala, S., Murray, H. W., Fortune, M. R., \& Barlow, D. H. (2013). Anxiety sensitivity and interoceptive exposure: A transdiagnostic construct and change strategy. Behavior Therapy, 44(3), 417-431. https://doi.org/10. 1016/j.beth.2013.03.006

Brake, C. A., Sauer-Zavala, S., Boswell, J. F., Gallagher, M. W., Farchione, T. J., \& Barlow, D. H. (2016). Mindfulness-based exposure strategies as a transdiagnostic mechanism of change: An exploratory alternating treatment design. Behavior Therapy, 47(2), 225-238. https://doi.org/10.1016/j.beth.2015.10.008

Bullis, J. R., Boettcher, H., Sauer-Zavala, S., Farchione, T. J., \& Barlow, D. H. (2019). What is an emotional disorder? A transdiagnostic mechanistic definition with implications for assessment, treatment, and prevention. Clinical Psychology, 26(2), e12278. https://doi.org/10.1111/cpsp.12278

Cassiello-Robbins, C., Southward, M. W., Tirpak, J. W., \& Sauer-Zavala, S. (2020). A systematic review of unified protocol applications with adult populations: Facilitating widespread dissemination via adaptability. Clinical Psychology Review, 78, 101852. https://doi.org/10.1016/j.cpr.2020.101852

Chadwick, P., Hember, M., Symes, J., Peters, E., Kuipers, E., \& Dagnan, D. (2008). Responding mindfully to unpleasant thoughts and images: Reliability and validity of the Southampton mindfulness questionnaire (SMQ). The British Journal of Clinical Psychology, 47(Pt 4), 451-455. https://doi.org/10.1348/014466508X314891

Constantino, M. J., Vîslă, A., Coyne, A. E., \& Boswell, J. F. (2018). A meta-analysis of the association between patients' early treatment outcome expectation and their posttreatment outcomes. Psychotherapy, 55(4), 473-485. https://doi.org/10.1037/pst0000169

Cuijpers, P., Reijnders, M., \& Huibers, M. J. H. (2019). The role of common factors in psychotherapy outcomes. Annual Review of Clinical Psychology, 15(1), 207-231. https://doi.org/10.1146/ annurev-clinpsy-050718-095424

Curreri, A. J., Farchione, T. J., Sauer-Zavala, S., \& Barlow, D. H. (2020). Mindful emotion awareness facilitates engagement with exposure therapy: An idiographic exploration using single case experimental design. Behavior Modification. https://doi.org/10. 1177/0145445520947662 
Dalgleish, T., Black, M., Johnston, D., \& Bevan, A. (2020). Transdiagnostic approaches to mental health problems: Current status and future directions. Journal of Consulting and Clinical Psychology, 88(3), 179-195. https://doi.org/10.1037/ccp0000482

Derogatis, L. R. (2000). BSI-18: Brief Symptom Inventory 18-Administration, scoring, and procedures manual. NCS Pearson.

Derogatis, L. R., \& Fitzpatrick, M. (2004). The SCL-90-R, the Brief Symptom Inventory (BSI), and the BSI-18. In The use of psychological testing for treatment planning and outcomes assessment: Instruments for adults. Lawrence Erlbaum Associates Publishers.

DeRubeis, R. J., Gelfand, L. A., German, R. E., Fournier, J. C., \& Forand, N. R. (2014). Understanding processes of change: How some patients reveal more than others - and some groups of therapists less-about what matters in psychotherapy. Psychotherapy Research, 24(3), 419-428. https://doi.org/10.1080/10503307. 2013.838654

Domhardt, M., Steubl, L., Boettcher, J., Buntrock, C., Karyotaki, E., Ebert, D. D., Cuijpers, P., \& Baumeister, H. (2020). Mediators and mechanisms of change in internet- and mobile-based interventions for depression: A systematic review. Clinical Psychology Review. https://doi.org/10.1016/j.cpr.2020.101953

El Alaoui, S., Ljótsson, B., Hedman, E., Kaldo, V., Andersson, E., Rück, C., Andersson, G., \& Lindefors, N. (2015). Predictors of symptomatic change and adherence in internet-based cognitive behaviour therapy for social anxiety disorder in routine psychiatric care. PLoS ONE, 10(4), e0124258. https://doi.org/10.1371/journ al.pone. 0124258

El Alaoui, S., Ljótsson, B., Hedman, E., Svanborg, C., Kaldo, V., \& Lindefors, N. (2016). Predicting outcome in internet-based cognitive behaviour therapy for major depression: A large cohort study of adult patients in routine psychiatric care. PLOS ONE, 11(9), e0161191. https://doi.org/10.1371/journal.pone.0161191

Ellard, K. K., Fairholme, C. P., Boisseau, C. L., Farchione, T. J., \& Barlow, D. H. (2010). Unified protocol for the transdiagnostic treatment of emotional disorders: Protocol development and initial outcome data. Cognitive and Behavioral Practice, 1, 88-101. https://doi.org/10.1016/j.cbpra.2009.06.002

Eustis, E. H., Cardona, N., Nauphal, M., Sauer-Zavala, S., Rosellini, A. J., Farchione, T. J., \& Barlow, D. H. (2019). Experiential avoidance as a mechanism of change across cognitive-behavioral therapy in a sample of participants with heterogeneous anxiety disorders. Cognitive Therapy and Research, 135(4), 166. https:// doi.org/10.1007/s10608-019-10063-6

Eustis, E. H., Hayes-Skelton, S. A., Roemer, L., \& Orsillo, S. M. (2016). Reductions in experiential avoidance as a mediator of change in symptom outcome and quality of life in acceptancebased behavior therapy and applied relaxation for generalized anxiety disorder. Behaviour Research and Therapy, 87, 188-195. https://doi.org/10.1016/j.brat.2016.09.012

Farchione, T. J., Fairholme, C. P., Ellard, K. K., Boisseau, C. L., Thompson-Hollands, J., Carl, J. R., Gallagher, M. W., \& Barlow, D. H. (2012). Unified protocol for transdiagnostic treatment of emotional disorders: A randomized controlled trial. Behavior Therapy, 43(3), 666-678. https://doi.org/10.1016/j.beth.2012.01. 001

Fisher, A. J., Bosley, H. G., Fernandez, K. C., Reeves, J. W., Soyster, P. D., Diamond, A. E., \& Barkin, J. (2019). Open trial of a personalized modular treatment for mood and anxiety. Behaviour research and therapy. Behaviour Research and Therapy, 116, 69-79. https://doi.org/10.1016/j.brat.2019.01.010

Flückiger, C., Del Re, A. C., Wampold, B. E., \& Horvath, A. O. (2018). The alliance in adult psychotherapy: A meta-analytic synthesis. Psychotherapy, 55(4), 316. https://doi.org/10.1037/pst0000172

Franke, G. H., Ankerhold, A., Haase, M., Jäger, S., Tögel, C., Ulrich, C., \& Frommer, J. (2011). Der Einsatz des Brief Symptom Inventory 18 (BSI-18) bei Psychotherapiepatienten. Psychotherapie,
Psychosomatik, Medizinische Psychologie, 61(2), 82-86. https:// doi.org/10.1055/s-0030-1270518

Fuhr, K., Hautzinger, M., Krisch, K., Berking, M., \& Ebert, D. D. (2016). Validation of the behavioral activation for depression scale (BADS)-Psychometric properties of the long and short form. Comprehensive Psychiatry, 66, 209-218. https://doi.org/ 10.1016/j.comppsych.2016.02.004

Gámez, W., Chmielewski, M., Kotov, R., Ruggero, C., Suzuki, N., \& Watson, D. (2014). The brief experiential avoidance questionnaire: Development and initial validation. Psychological Assessment, 26(1), 35-45. https://doi.org/10.1037/a0034473

Goldin, P. R., Morrison, A., Jazaieri, H., Brozovich, F., Heimberg, R., \& Gross, J. J. (2016). Group CBT versus MBSR for social anxiety disorder: A randomized controlled trial. Journal of Consulting and Clinical Psychology, 84(5), 427-437. https://doi.org/10.1037/ cсp0000092

Gross, J. J., \& John, O. P. (2003). Individual differences in two emotion regulation processes: Implications for affect, relationships, and well-being. Journal of Personality and Social Psychology, 85(2), 348-362. https://doi.org/10.1037/0022-3514.85.2.348

Hofmann, S. G., Curtiss, J. E., \& Hayes, S. C. (2020). Beyond linear mediation: Toward a dynamic network approach to study treatment processes. Clinical Psychology Review, 76, 101824. https:// doi.org/10.1016/j.cpr.2020.101824

Hofmann, S. G., \& Hayes, S. C. (2019). The future of intervention science: Process-based therapy. Clinical Psychological Science, 7(1), 37-50. https://doi.org/10.1177/2167702618772296

Kazantzis, N., Luong, H. K., Usatoff, A. S., Impala, T., Yew, R. Y., \& Hofmann, S. G. (2018). The processes of cognitive behavioral therapy: A review of meta-analyses. Cognitive Therapy and Research, 42(4), 349-357. https://doi.org/10.1007/ s10608-018-9920-y

Kazdin, A. E. (2007). Mediators and mechanisms of change in psychotherapy research. Annual Review of Clinical Psychology, 3, 1-27. https://doi.org/10.1146/annurev.clinpsy.3.022806.091432

Kemper, C. J., Ziegler, M., \& Taylor, S. (2009). Überprüfung der psychometrischen Qualität der deutschen Version des Angstsensitivitätsindex-3. Diagnostica, 55(4), 223-233. https://doi.org/10. 1026/0012-1924.55.4.223

Kocovski, N. L., Fleming, J. E., Hawley, L. L., Ho, M.-H.R., \& Antony, M. M. (2015). Mindfulness and acceptance-based group therapy and traditional cognitive behavioral group therapy for social anxiety disorder: Mechanisms of change. Behaviour Research and Therapy, 70, 11-22. https://doi.org/10.1016/j.brat.2015.04.005

Kuznetsova, A., Brockhoff, P. B., \& Christensen, R. H. B. (2017). ImerTest package: Tests in linear mixed effects models. Journal of Statistical Software. https://doi.org/10.18637/jss.v082.i13

Lemmens, L. H. J. M., Müller, V. N. L. S., Arntz, A., \& Huibers, M. J. H. (2016). Mechanisms of change in psychotherapy for depression: An empirical update and evaluation of research aimed at identifying psychological mediators. Clinical Psychology Review, 50, 95-107. https://doi.org/10.1016/j.cpr.2016.09.004

Manos, R. C., Kanter, J. W., \& Luo, W. (2011). The behavioral activation for depression scale-short form: Development and validation. Behavior Therapy, 42(4), 726-739. https://doi.org/10.1016/j.beth. 2011.04.004

Mansell, W., \& McEvoy, P. M. (2017). A test of the core process account of psychopathology in a heterogenous clinical sample of anxiety and depression. A case of the blind men and the elephant? Journal of Anxiety Disorders, 46, 4-10.

Margraf, J., Cwik, J. C., Suppiger, A., \& Schneider, S. (2017). DIPS open access: Diagnostic interview for mental disorders. [DIPS Open Access: Diagnostisches Interview bei psychischen Störungen.]. Mental Health Research and Treament Center, Ruhr-Universität Bochum. 
Newby, J. M., McKinnon, A., Kuyken, W., Gilbody, S., \& Dalgleish, T. (2015). Systematic review and meta-analysis of transdiagnostic psychological treatments for anxiety and depressive disorders in adulthood. Clinical Psychology Review, 40, 91-110. https://doi. org/10.1016/j.cpr.2015.06.002

Păsărelu, C. R., Andersson, G., Bergman Nordgren, L., \& Dobrean, A. (2017). Internet-delivered transdiagnostic and tailored cognitive behavioral therapy for anxiety and depression: A systematic review and meta-analysis of randomized controlled trials. Cognitive Behaviour Therapy, 46(1), 1-28. https://doi.org/10.1080/ 16506073.2016.1231219

Pearl, S. B., \& Norton, P. J. (2017). Transdiagnostic versus diagnosis specific cognitive behavioural therapies for anxiety: A meta-analysis. Journal of Anxiety Disorders, 46, 11-24. https://doi.org/10. 1016/j.janxdis.2016.07.004

Preacher, K. J., \& Hayes, A. F. (2008). Asymptotic and resampling strategies for assessing and comparing indirect effects in multiple mediator models. Behavior Research Methods, 40(3), 879-891. https://doi.org/10.3758/BRM.40.3.879

Preacher, K. J., \& Kelley, K. (2011). Effect size measures for mediation models: Quantitative strategies for communicating indirect effects. Psychological Methods, 16(2), 93-115. https://doi.org/10. 1037/a0022658

Prinz, U., Nutzinger, D. O., Schulz, H., Petermann, F., Braukhaus, C., \& Andreas, S. (2013). Comparative psychometric analyses of the SCL-90-R and its short versions in patients with affective disorders. BMC Psychiatry, 13, 104. https://doi.org/10.1186/ 1471-244X-13-104

Probst, G. H., Berger, T., \& Flückiger, C. (2019). The alliance-outcome relation in internet-based interventions for psychological disorders: A correlational meta-analysis. Verhaltenstherapie. https:// doi.org/10.1159/000503432

Rosseel, Y. (2012). lavaan: An R package for structural equation modeling. Journal of Statistical Software. https://doi.org/10.18637/ jss.v048.i02

Rozental, A., Andersson, G., \& Carlbring, P. (2019). In the absence of effects: An individual patient data meta-analysis of non-response and its predictors in internet-based cognitive behavior therapy. Frontiers in Psychology. https://doi.org/10.3389/fpsyg.2019. 00589

RStudio Team. (2019). RStudio: Integrated Development Environment for $R$. RStudio, Inc. http://www.rstudio.com/

Sakiris, N., \& Berle, D. (2019). A systematic review and meta-analysis of the Unified Protocol as a transdiagnostic emotion regulation based intervention. Clinical Psychology Review, 72, 101751. https://doi.org/10.1016/j.cpr.2019.101751

Sauer-Zavala, S., Cassiello-Robbins, C., Ametaj, A. A., Wilner, J. G., \& Pagan, D. (2018). Transdiagnostic treatment personalization: The feasibility of ordering unified protocol modules according to patient strengths and weaknesses. Behavior Modification. https:// doi.org/10.1177/0145445518774914

Sauer-Zavala, S., Cassiello-Robbins, C., Conklin, L. R., Bullis, J. R., Thompson-Hollands, J., \& Kennedy, K. A. (2017a). Isolating the unique effects of the unified protocol treatment modules using single case experimental design. Behavior Modification, 41(2), 286-307. https://doi.org/10.1177/0145445516673827
Sauer-Zavala, S., Gutner, C. A., Farchione, T. J., Boettcher, H. T., Bullis, J. R., \& Barlow, D. H. (2017b). Current definitions of "transdiagnostic" in treatment development: A search for consensus. Behavior Therapy, 48, 128-138. https://doi.org/10.1016/j.beth. 2016.09.004

Schaeuffele, C., Homeyer, S. L., Perea, L., Scharf, L., Schulz, A., Knaevelsrud, C., Renneberg, B., \& Boettcher, J. (2020). The unified protocol as an internet-based intervention for emotional disorders: Randomized controlled trial [Preprint]. PsyArXiv. https:// doi.org/10.31234/osf.io/528tw

Schaeuffele, C., Knaevelsrud, C., Renneberg, B., \& Boettcher, J. (2021a). Psychometric properties of the German Brief Experiential Avoidance Questionnaire (BEAQ). Assessment, 107319112110109. https://doi.org/10.1177/10731911211010955

Schaeuffele, C., Schulz, A., Knaevelsrud, C., Renneberg, B., \& Boettcher, J. (2021b). CBT at the crossroads: The rise of transdiagnostic treatments. International Journal of Cognitive Therapy, 14(1), 86-113. https://doi.org/10.1007/s41811-020-00095-2

Southward, M. W., Sauer-Zavala, S., \& Cheavens, J. S. (2021). Specifying the mechanisms and targets of emotion regulation: A translational framework from affective science to psychological treatment. Clinical Psychology. https://doi.org/10.1037/cps0000003

Spinhoven, P., van Hemert, A. M., \& Penninx, B. W. J. H. (2017). Experiential avoidance and bordering psychological constructs as predictors of the onset, relapse and maintenance of anxiety disorders: One or Many? Cognitive Therapy and Research, 41(6), 867-880. https://doi.org/10.1007/s10608-017-9856-7

Taylor, S., Zvolensky, M. J., Cox, B. J., Deacon, B., Heimberg, R. G., Ledley, D. R., Abramowitz, J. S., Holaway, R. M., Sandin, B., Stewart, S. H., Coles, M., Eng, W., Daly, E. S., Arrindell, W. A., Bouvard, M., \& Cardenas, S. J. (2007). Robust dimensions of anxiety sensitivity: Development and initial validation of the Anxiety Sensitivity Index-3. Psychological Assessment, 19(2), 176-188. https://doi.org/10.1037/1040-3590.19.2.176

Valente, M. J., \& MacKinnon, D. P. (2017). Comparing models of change to estimate the mediated effect in the pretest-posttest control group design. Structural Equation Modeling, 24(3), 428-450. https://doi.org/10.1080/10705511.2016.1274657

Wampold, B. E. (2015). How important are the common factors in psychotherapy? An Update. World Psychiatry, 14(3), 270-277. https://doi.org/10.1002/wps.20238

Wen, Z., \& Fan, X. (2015). Monotonicity of effect sizes: Questioning kappa-squared as mediation effect size measure. Psychological Methods, 20(2), 193-203. https://doi.org/10.1037/met0000029

Woods, B. K., Sauer-Zavala, S., Farchione, T. J., \& Barlow, D. H. (2020). Isolating the effects of mindfulness training across anxiety disorder diagnoses in the unified protocol. Behavior Therapy, 51(6), 972-983. https://doi.org/10.1016/j.beth.2020.01.001

Publisher's Note Springer Nature remains neutral with regard to jurisdictional claims in published maps and institutional affiliations. 